\title{
Tecnologia em enfermagem: análise conceitual à luz do modelo evolucionário de
}

\author{
Rodgers \\ Technology in nursing: conceptual analysis in the light of the evolutionary model of Rodgers \\ Tecnología en enfermería: análisis conceptual a la luz del modelo evolucionario de Rodgers
}

Recebido: 13/01/2022 | Revisado: 17/01/2022 | Aceito: 23/01/2022 | Publicado: 25/01/2022

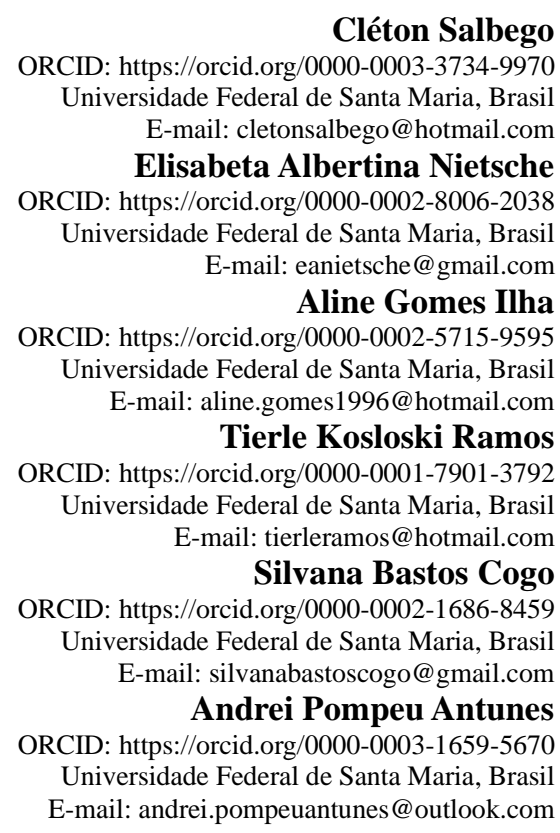

\section{Resumo}

O objetivo desse trabalho foi analisar a evolução do conceito de tecnologia em enfermagem empregado nas teses e dissertações brasileiras de enfermagem. Utilizou-se a análise conceitual, norteado pelo modelo evolucionário de Rodgers. Coleta de dados de fevereiro a março de 2019, mediante consulta aos relatórios depositados no Banco de Teses e Dissertações da Coordenação de Aperfeiçoamento de Pessoal de Nível Superior entre 2005 a 2018 e, posteriormente a captação, houve análise descritiva. Os antecedentes e consequentes remeteram a integralidade no cuidado, tecnologia, informação, globalização e prática clínica em enfermagem. Os termos substitutos associam-se ao conceito de interesse. Os conceitos relacionados foram os apresentados por pesquisadores como Merhy e Nietsche. Os atributos do conceito podem ser categorizados como gerais. Concluiu-se que a compreensão sobre o conceito de tecnologia evoluiu teoricamente e na aplicação na prática de enfermagem. O conceito perpassou a atuação do enfermeiro frente à gerência, educação e assistência direta ou indiretamente relacionadas ao cuidado.

Palavras-chave: Tecnologia biomédica; Tecnologia; Formação de conceito; Enfermagem.

\begin{abstract}
The objective of this study was to analyze the evolution of the concept of technology in nursing used in Brazilian nursing theses and dissertations. Conceptual analysis was used, guided by Rodgers' evolutionary model. Data collection from February to March 2019, by consulting the reports deposited in the Bank of Theses and Dissertations of the Coordination for the Improvement of Higher Education Personnel between 2005 and 2018 and, after the capture, there was a descriptive analysis. The antecedents and consequences referred to integrality in care, technology, information, globalization and clinical practice in nursing. Substitute terms are associated with the concept of interest. Related concepts were those presented by researchers such as Merhy and Nietsche. Concept attributes can be categorized as general. It was concluded that the understanding of the concept of technology has evolved theoretically and in application in nursing practice. The concept permeated the nurse's role in management, education and assistance directly or indirectly related to care.
\end{abstract}

Keywords: Biomedical technology; Technology; Concept formation; Nursing.

\section{Resumen}

El objetivo de este trabajo fue analizar la evolución del concepto de tecnología en enfermería utilizado en las tesis y disertaciones de enfermería brasileñas. Se utilizó el análisis conceptual, guiado por el modelo evolutivo de Rodgers. 
Recolección de datos de febrero a marzo de 2019, mediante consulta de los informes depositados en el Banco de Tesis y Disertaciones de la Coordinación para el Perfeccionamiento del Personal de Educación Superior entre 2005 y 2018 y, posterior a la captura, se realizó un análisis descriptivo. Los antecedentes y consecuencias referidos a la integralidad en el cuidado, tecnología, información, globalización y práctica clínica en enfermería. Los términos sustitutivos están asociados con el concepto de interés. Conceptos relacionados fueron los presentados por investigadores como Merhy y Nietzsche. Los atributos del concepto se pueden categorizar como generales. Se concluyó que la comprensión del concepto de tecnología ha evolucionado teóricamente y en la aplicación en la práctica de enfermería. El concepto permeó el papel del enfermero en la gestión, educación y asistencia directa o indirectamente relacionada con el cuidado.

Palabras clave: Tecnología biomédica; Tecnología; Formación de conceptos; Enfermería.

\section{Introdução}

Um conceito é uma ideia ou construção mental elaborada acerca de um fenômeno (Poles et al., 2009). São termos referentes aos fenômenos que ocorrem na natureza ou no pensamento (Mecwen et al., 2009). São representações cognitivas, abstratas, de uma realidade perceptível formada por experiências diretas ou indiretas. Os conceitos podem ser empíricos ou concretos (observados pelos sentidos) ou abstratos (não observáveis) e sua função primária é permitir que indivíduos possam descrever situações e se comunicar efetivamente (Morse, 1995). Nesta perspectiva, "esse termo tem significado generalíssimo e pode incluir qualquer espécie de sinal ou procedimento semântico, seja qual for o objeto a que se refere, abstrato ou concreto, próximo ou distante, universal ou individual" (Abbagnano, 2007, p.164).

Para desenvolver uma análise conceitual, se faz necessário a revisão dos atributos essenciais que são reavaliados por meio de deliberações destes conceitos por intermédio da análise conceitual, método que compõe uma investigação completa sobre fundamentos substanciais que fazem parte de um pensamento, ideia ou discernimento (Burnell, 2009). Sob esse enfoque, recomenda-se que a análise deva ser realizada no momento em que um conceito já colocado, definido e explicado na literatura de uma disciplina, sendo indispensável a adição de uma nova pesquisa para elevá-lo a um determinado nível de desenvolvimento, de maneira a aplicá-lo produtivamente no estudo e na prática dessas disciplinas, acrescendo sua percepção entre o público que o utiliza (Mecwen et al., 2009).

Pensar em tecnologia é considerá-la, como um conjunto de saberes e conhecimentos científicos e sistematizados, a partir da pesquisa e da experiência cotidiana dos profissionais, pois estas possibilitam ao profissional pensar, refletir e agir ao ponto de torná-lo ser ativo do seu processo de existência. Há que se destacar que verificar a tecnologia sob uma perspectiva de construção, a partir da experiência dos profissionais de enfermagem nos diferentes cenários, implica em introduzir neste campo do trabalho estratégias (processos), e materialidades (produtos) que possam atender as necessidades advindas da avaliação e reflexão frente a demandas identificadas no cotidiano de trabalho da disciplina (Nietsche et al., 2012; Nietsche et al., 2021).

Historicamente este conceito vem sendo associado a construção, utilização e validação/avaliação de tecnologias de produto. Nesta perspectiva, estudo que analisou a práxis de enfermeiros frente ao uso destas ferramentas, atribuiu predominantemente a utilização das máquinas para o desenvolvimento do cuidado em enfermagem (Salbego et al., 2018; Salbego, Nietsche, Ramos, et al. 2020).

Desse modo, essa pesquisa justifica-se por promover um estudo com ênfase nas tecnologias, e analisar de que modo este termo está sendo compreendido/concebido no âmbito da enfermagem, ao longo dos anos, e por saber que neste cenário de atuação concentram-se recursos e aparatos altamente tecnológicos, que por vezes, são associados de modo reducionista a máquina (produto). Delineou-se como questão de pesquisa: qual a evolução do conceito de tecnologia empregado nas produções científicas de enfermagem? Portanto, objetivou-se analisar a evolução do conceito de tecnologia em enfermagem empregado nas teses e dissertações brasileiras de enfermagem. 


\section{Metodologia}

Estudo de análise conceitual, que utilizou como método o modelo evolucionário proposto por Rodgers (Rodgers, 2000). Realizado a partir do mapeamento das produções dos Programas stricto senso de Pós-Graduação em Enfermagem no Brasil que utilizaram o conceito de tecnologia em enfermagem. A análise de conceito no método evolucionário de Rodgers (2000) propõe um processo interativo em toda a investigação, permeado por seis etapas: (1) identificação do conceito de interesse e as expressões associadas; (2) identificação e seleção do campo apropriado para a coleta de dados; (3) coleta dos dados relevantes para a análise do conceito; (4) analise dos dados e identificação das características do conceito; (5) identificação de um exemplo do conceito; e (6) identificação de hipóteses e implicações para o desenvolvimento futuro do conceito (Rodgers et al., 2000).

Correspondendo a primeira etapa, delimitou-se como conceito de interesse o termo "tecnologia em enfermagem" empregado nas produções científicas de enfermagem, bem como, suas expressões associadas. Na segunda etapa, a identificação e seleção do campo para coleta de dados, utilizou-se o Banco de Teses e Dissertações da Coordenação de Aperfeiçoamento de Pessoal de Nível Superior (CAPES). A referida escolha deu-se pelo fato dessa fonte abarcar, em sua totalidade as produções de dissertações e teses de enfermagem na pesquisa científica, modalidade esta, de trabalhos caracterizadas pela variância temática, criatividade, rigorosidade e logicidade, elementos pertinentes para o desenvolvimento de pesquisas.

A coleta de dados, realizada entre fevereiro e março de 2019, utilizando a palavra-chave "tecnologia", obteve-se a localização de 797 pesquisas. Para a seleção adotou-se os, seguintes critérios de inclusão: (1) ser dissertações ou teses; (2) estar vinculado aos Programas de Pós-Graduação em Enfermagem do Brasil; (3) ser pesquisa que aborde tecnologias em enfermagem; (4) recorte temporal de 2005 a 2018; e (5) ter o conceito de tecnologia claramente descrito no desenvolvimento da pesquisa. Há que se destacar que o recorte temporal atribuído, deu-se em virtude do aumento da produção científica na enfermagem, com reflexões a respeito dos modelos assistenciais, gerenciais, educacionais e tecnológicos. Excluíram-se estudos que não estavam disponíveis eletronicamente na integra no banco de dados da CAPES e/ou repositórios das Instituições de Ensino Superior (IES) de origem.

O desenvolvimento da terceira e quarta etapas foram norteados pelos seguintes questionamentos (Quadro 1):

Quadro 1 - Elementos analíticos e questionamentos sobre a análise conceitual do termo tecnologia à luz do método Evolucionário de Rodgers.

\begin{tabular}{|c|l|}
\hline Elementos Analíticos & \multicolumn{1}{c|}{ Questionamentos } \\
\hline Antecedentes & Quais eventos/fenômenos contribuíram para o surgimento do termo? \\
\hline Consequentes & Quais as consequências após a aplicação do termo? \\
\hline Termos substitutos & Quais as palavras ou expressões que substituem o termo? \\
\hline Conceitos relacionados & Quais suposições filosóficas que formulam o significado do termo? \\
\hline Atributos/conceitos & $\begin{array}{l}\text { Como os pesquisadores conceituam tecnologia em enfermagem? } \\
\text { Quais as principais características apresentadas pelos pesquisadores acerca do termo? }\end{array}$ \\
\hline
\end{tabular}

Fonte: Adaptado de Rodgers (2000).

Para captação dos estudos, foi realizada a leitura dos títulos e resumos dos 797 estudos, e, obteve-se um total de 176 produções abordaram a temática de tecnologias na área da enfermagem. Posteriormente, foi realizada a leitura na integra, onde 
98 pesquisas não apresentaram o conceito de interesse claramente descrito, resultando em 78 produções, destas, 59 dissertações de mestrado e 19 teses de doutorado, as quais proporcionaram concretizar a análise contextual do conceito.

Os dados foram organizados em ordem cronológica em uma planilha do Microsoft Office Excel® 2013, de acordo com identificação do estudo, o ano de publicação, objetivo (s), características metodológicas, antecedentes, consequentes, termos substitutos, conceitos relacionados e atributos/conceitos.

A fim de favorecer a análise, os dados das teses e dissertações foram apresentados em conjunto. Complementa-se, ainda, que esta pesquisa se isenta do Termo de Consentimento Livre e Esclarecido (TCLE) por seu caráter documental. Como os documentos analisados nesta pesquisa foram extraídos de dados públicos, os aspectos éticos foram respeitados, considerando que as informações expostas traduzem o que tratam as pesquisas.

\section{Resultados}

Os resultados foram organizados de maneira indutiva, baseados na proposição de Rodgers (2000) que considera os antecedentes, consequentes, termos substitutivos, conceitos relacionados e atributos do conceito.

\subsection{Antecedentes e consequentes do conceito}

A partir da análise das produções os antecedentes partem de um acontecimento ou fenômeno específico. Assim, os estudos apontaram para as necessidades enfrentadas pelos usuários/pacientes frente ao processo de saúde-adoecimento. Também, revelaram o processo criativo dos enfermeiros a partir da produção tecnológica com vistas a resolução de demandas assistenciais, gerenciais e educacionais.

Resultante a estes antecedentes, identificou-se os consequentes, que se apresentaram voltados à melhoria da assistência prestada pelos profissionais de enfermagem (Figura 1).

Figura 1 - Antecedentes e consequentes do conceito de tecnologia empregado na produção científica de enfermagem.

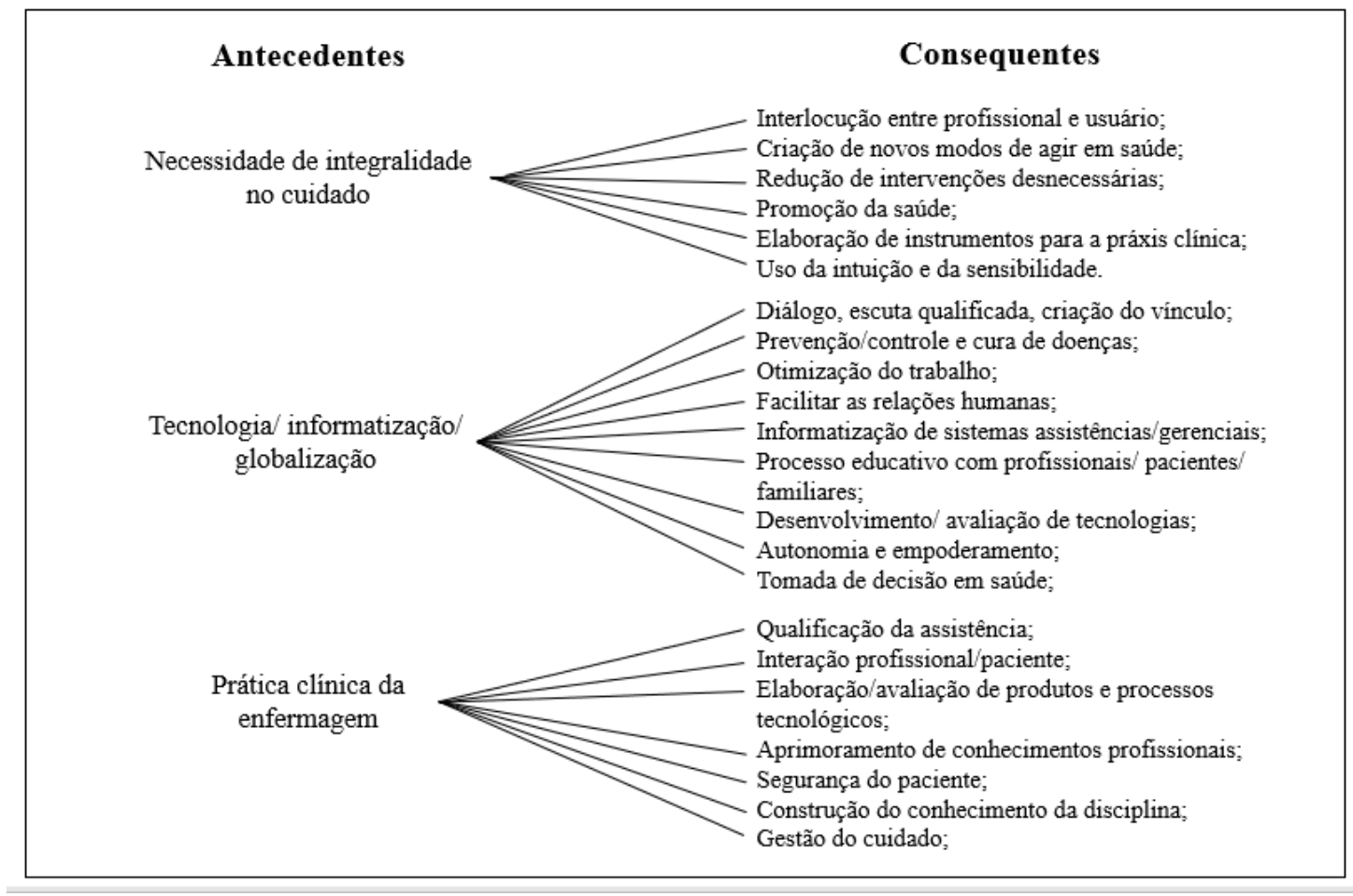

Fonte: Autores. 


\subsection{Termos substitutos, conceitos relacionados e atributos do conceito}

Os termos substitutos associam-se com o conceito de tecnologia em enfermagem presente no estudo e com a inserção da referida tecnologia na prática dos profissionais. Já os conceitos relacionados, são suposições filosóficas que formulam o significado do termo tecnologia. E os atributos do conceito dizem respeito a maneira como os pesquisadores conceituam tecnologia e as principais características apresentadas acerca do termo (Figura 2).

Figura 2 - Termos substitutos, conceitos relacionados e atributos do conceito de tecnologia na produção científica de enfermagem.

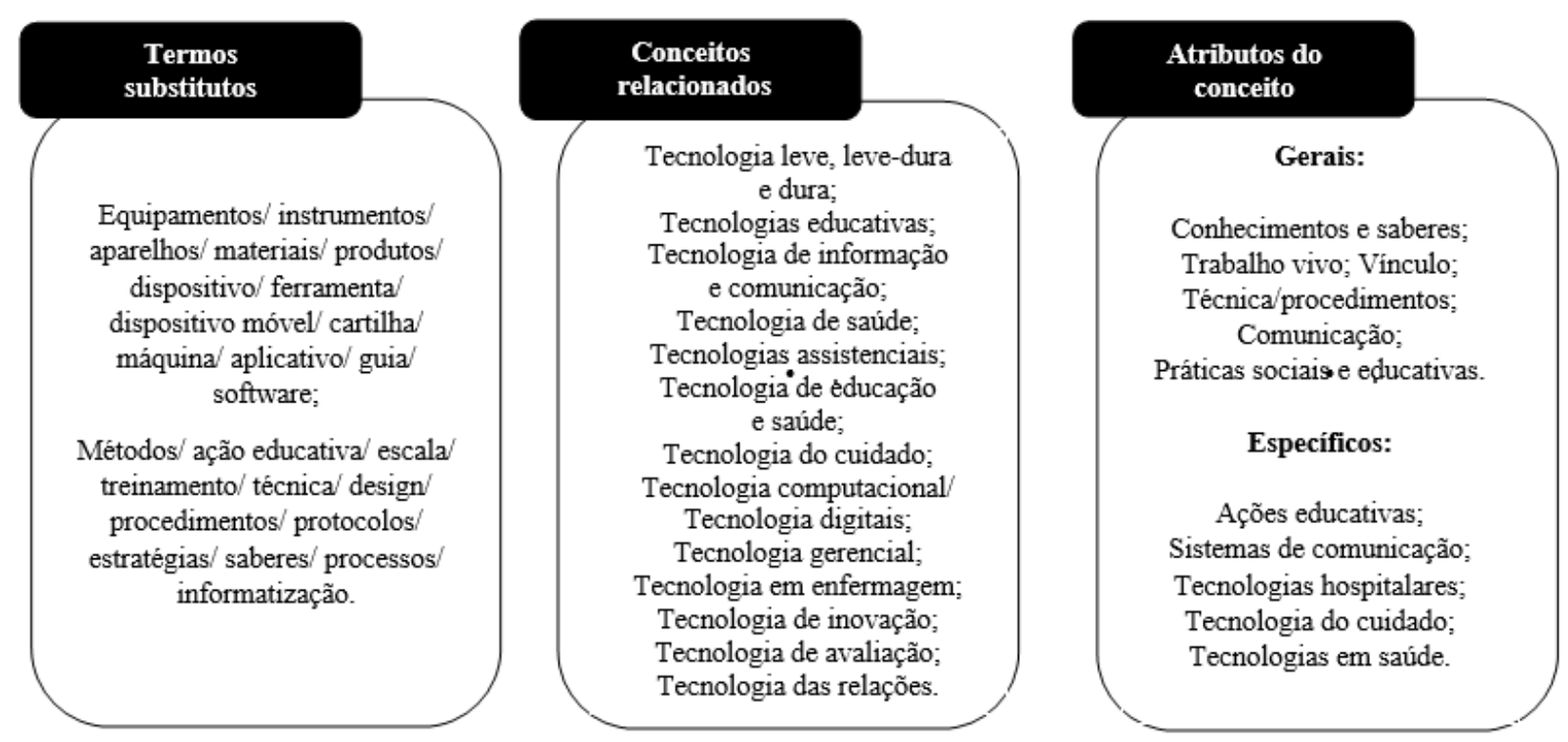

Fonte: Autores.

\section{Discussão}

Em relação aos antecedentes e consequentes do conceito tecnologia em enfermagem, destaca-se a premência da revolução industrial, pois trouxe consigo um conjunto de transformações tecnológicas que proporcionaram mudanças para a assistência em saúde (Nietsche et al., 2012). Para tanto, registra-se que o desenvolvimento técnico-científico da humanidade, obteve nas guerras e lutas de dominação entre os seres humanos uma motivação preponderante. Com a ocorrência de duas guerras mundiais no século XX, além de gerar uma corrida desenfreada pelo desenvolvimento de tecnologias para a destruição, também de forma ambivalente, estas também têm servido para a melhoria da vida das pessoas (Nietsche et al., 2021).

Também é atribuído ao crescimento da tecnologia o progresso e a evolução histórica vivenciada pela sociedade. No universo de atuação da enfermagem, as transformações do cuidado são progressivamente influenciadas pelo avanço da ciência e da tecnologia, o que têm resultando em certas inquietações e questionamentos em relação aos seus benefícios, riscos e vínculos construídos entre trabalhadores, pacientes e a atribuição de tecnologias como instrumento indispensável ao cuidado de enfermagem/saúde (Schwonke et al., 2011; Nietsche et al., 2021).

Nesta perspectiva a avaliação de tecnologias em saúde torna-se importante, como um processo contínuo de análise dos benefícios para a saúde, das consequências econômicas e sociais, ao considerar os seguintes aspectos: segurança, eficácia, efetividade, custos, custo-efetividade e equidade, impactos éticos, culturais e ambientais. Assim, o objetivo é auxiliar as instâncias decisórias quanto à utilização e inspeção de tecnologias nos estabelecimentos de saúde, além de informar aos trabalhadores de saúde e clientes em relação à segurança, aos benefícios e aos custos dos recursos (Brasil, 2010). 
A tecnologia transforma a maneira de viver e é determinante em sua influência em diversos campos, social, econômico e ambiental, interferindo no estilo de vida, desenvolvendo controle cultural e social sobre o ser humano (Silva et al., 2011; Nietsche et al., 2021). Desta maneira a revolução tecnológica, trouxe influências diretas para a atuação da enfermagem, proporcionando maior qualidade, eficácia, efetividade e segurança ao cuidado. Quando atrelada ao conhecimento científico e técnico possibilita condições contribuintes a qualidade de vida. Frente ao apresentado, conclui-se que o uso das tecnologias no cotidiano da enfermagem, possibilita o desenvolver de um cuidado ético, humanizado e seguro (Trbovich et al., 2010; Salbego et al., 2018).

A constante aplicação das tecnologias nos cenários de atuação da enfermagem contribuiu para o aprimoramento do cuidado em três esferas: assistência, gerência e educação. Igualmente nas relações interpessoais instauradas entre diferentes sujeitos. O emprego das tecnologias é influenciado diretamente pela significação atribuída durante sua concepção e utilização como ferramenta de cuidado (Nietsche et al., 2012; Salbego et al., 2021). Frente ao exposto, as tecnologias em enfermagem almejam a eficiência das atividades nas diversas esferas, e para isso ela produz distintos objetos para suprir às necessidades ou aprimorar os recursos tornando-os mais resistentes (Koerich, 2006, Nietsche et al., 2021).

Em relação aos termos substitutos, foi possível inferir que as tecnologias em enfermagem são relacionadas a produtos e processos. As tecnologias como produto devem conceber reflexões críticas relacionadas à importância de sua elaboração, objetivo e público alvo. Não é possível desligar os produtos, máquinas/ferramentas, de um trabalho vivo, ou seja, da atuação do profissional, ação e/ou expressão, junto a esta tecnologia (Aquino et al., 2010). Além da tecnologia como produto, com menor destaque, aparece a tecnologia como processo, que para ser utilizada, deve apresentar as fases delimitadas em sua elaboração (Aquino et al., 2010). De modo geral, observa-se que os estudos se utilizam dos conceitos de tecnologia, apresentados por pesquisadores como Merhy e Nietsche, referenciadas internacionalmente na temática.

Em relação aos conceitos relacionados, destacam-se as diferentes tipologias de tecnologias que surgem a partir da necessidade dos profissionais. Sendo assim, evidencia-se a progressiva utilização de recursos tecnológicos voltado aos cuidados em saúde e em enfermagem. Esta disseminação de recursos na enfermagem coopera para que a prática prospere, contribuindo para a modernização da enfermagem frente ao cuidado. $\mathrm{O}$ uso da tecnologia pela disciplina possibilita a consolidação da profissão enquanto ciência aplicada assegurando que novos saberes organizem-se, servindo de alicerce para a educação, pesquisa, prática e gerenciamento em enfermagem (Nietsche et al., 2020; Salbego, Nietsche, Ramos, 2021).

Quanto ao conceito de tecnologias, existem concepções de diferentes autores referentes ao termo, desde uma visão restrita e reducionista, que se relaciona ao produto/máquina, até uma visão ampla, que compreende saberes construídos pelos seres humanos. A palavra tecnologia é presença constante em atividades assistenciais, gerenciais e educativas da enfermagem, permitindo assim, inúmeras interpretações.

Frente ao exposto, o conceito de Merhy é o mais utilizado na atualidade, no que diz respeito ao conceito tecnológico. Para o autor supracitado a tecnologia pode ser classificada em leve, leve-dura e dura. Entretanto, independente da classificação, as tecnologias, necessitam de um acompanhamento do trabalho vivo, para efetividade do serviço em saúde. As tecnologias leves podem ser processos de produção da comunicação, leve-duras saberes estruturados nas disciplinas que atuam na área da saúde e duras materiais e equipamentos (Merhy, 2002).

Nietsche propõe um novo conceito, em que a tecnologia em enfermagem emerge por meio de um conjunto de conhecimentos/saberes ordenados, que geram processos concretizados a partir das vivências dos indivíduos e da pesquisa. Assim as tecnologias em enfermagem podem ser entendidas como intercessoras da racionalidade e subjetividade, intuição e emoções, resultando no crescimento e qualificação do cuidado a partir da razão e da sensibilidade (Nietsche et al., 2012). 
A partir das exposições dos principais autores supracitados durante os estudos, é importante ressaltar a amplitude conceitual de tecnologia apresentado, abandonando o conceito reducionista apenas ligado a máquinas, mas sim abrangendo seu entendimento a uma esfera global.

\section{Considerações Finais}

O conceito de tecnologia em enfermagem evoluiu ao longo dos anos, tendo como marco a revolução industrial que inseriu na área da saúde tecnologias de produto para subsidiar o cuidado. Neste sentido, foi possível associar uma evolução cronológica às concepções apresentadas.

A análise identificou que existem diferentes concepções para o termo, desde uma visão restrita e reducionista a produto/máquina, até uma visão ampliada, que compreende saberes construídos pelos seres humanos. As tecnologias em enfermagem estão presentes constantemente nas atividades assistenciais, gerenciais e educativas da enfermagem, permitindo assim, modernizar e qualificar o cuidado. Analisar a evolução deste termo permite aos profissionais enfermeiros desenvolverem estudos de produção, validação e aplicação de tecnologias sustentados por um conceito com características claras e coerentes.

\section{Referências}

Abbagnano, N. (2007). Dicionário de Filosofia. São Paulo: Revista e Ampliada.

Aquino, P. S.; Melo, R. P.; \& Lopes, M. V. O.; Pinheiro, A. K. B. (2010). Análise do conceito de tecnologia na enfermagem segundo o método evolucionário. Acta Paul Enferm, 23(5), 690-6.

Brasil. (2010). Ministério da Saúde. Secretaria de Ciência, Tecnologia e Insumos Estratégicos: Série B. Textos Básicos em Saúde, p.48. Brasília, DF.

Burnell, L (2009). Compassionate care: a concept analysis. Home health care manag. Pract, 21(5), 319-24.

Koerich, M. S.; Backes, D. S.; Scortegagna, H. M.; Wall, M. L.; Veronese, A. M.; Zeferino, M. T.; et al. (2006). Tecnologias de cuidado em saúde e enfermagem e suas perspectivas filosóficas. Texto Contexto Enferm, 15(Esp), 178-85.

Mcewen, M.; Wills, E. M. (2009). Bases teóricas para a enfermagem: os fundamentos à prática profissional. 2. Ed. Porto Alegre: Artmed.

Merhy, E. E. (2002). Saúde: a cartografia do trabalho vivo. 3. Ed. São Paulo: Hucitec.

Morse, J. M. (1995). Exploring the theoretical basis of nursing using advanced techniques of concept analysis. Adv Nurs Sci, 17(3), 31-46.

Nietsche, E. A.; Lima, M. G. R.; Rodrigues, M. G. S.; Teixeira, J. A.; Oliveira, B. N. B.; Motta, C. A.; et al. (2012). Tecnologias inovadoras do cuidado em enfermagem. Rev Enferm UFSM, 2(1), 182-189.

Nietsche, E. A., Cassenote, L. G., Salbego, C., Ramos, T. K., Perlini, NMOG, Böck, A., et al. (2020) Care Facilitator Cart: a product technology built with nursing professionals. Rev Bras Enferm. 73(Suppl 6), e20190741.

Nietsche, E. A.; Salbego, C.; Lacerda, M. R. (2021). Praxis and technological development in nursing. Rev. Enferm. UFSM.11(1), 1-3.

Poles, K.; Bousso, R. S. (2009). Morte digna da criança: análise de conceito. Rev Esc Enferm USP 43(1), $215-22$.

Rodgers, B. L.; Knafl, K. A. (2000). Introduction to concept development in nursing. In: Rogers, B. L.; Knafl, K. A.; editors. Concept development in nursing: foundations, techniques, and applications. 2. Ed. Philadelphia: Saunders, 1-6.

Rodgers, B. L. (2000). Concept analysis: an evolutionary view. In: Rogers, B. L.; Knafl, K. A.; editors. Concept development in nursing: foundations, techniques, and applications. 2. Ed. Philadelphia: Saunders, 77-102.

Salbego, C.; Nietsche, E. A.; Teixeira, E.; Girardon-Perlini, N. M. O.; Wild, C. F.; Ilha, S. (2018). Care-educational technologies: an emerging concept of the praxis of nurses in a hospital context. Rev Bras Enferm, 71(Suppl 6), 2666-74.

Salbego, C.; Nietsche, E. A.; Ramos, T. K.; et al. (2021) Concepções sobre tecnologias do cuidar e educar na práxis do enfermeiro hospitalar. Rev Fun Care Online. 13:150-157.

Schwonke, C. R. G. B.; Filho, W. D. L. L.; Lunardi, V. L.; Santos, S. S. C.; \& Barlem, E. L. D. (2011). Perspectivas filosóficas do uso da tecnologia no cuidado de enfermagem em terapia intensiva. Rev Bras Enferm, 64(1), 189-92.

Silva, R. C.; \& Ferreira, M. A. (2011). Tecnologia na terapia intensiva e suas influências nas ações do enfermeiro. Rev Esc Enferm USP, 45(6), 1403-11.

Trbovich, P. L.; Pinkney, S.; Cafazzo, J. A.; \& Easty, A. C. (2010). The impact of traditional and smart pump infusion technology on nurse medication administration performance in a simulated inpatient unit. Qual Saf Health Care, 19, 430-4. 